\title{
关于 $\bar{d}$ - 跟踪性质的一些注记
}

\section{吴新星}

电子科技大学数学学院, 成都 611731

E-mail: wuxinxing5201314@163.com

收稿日期: 2013-11-20；接受日期: 2014-09-16

四川省教育厅基金 (批准号: 14ZB0007) 和四川理工学院科研项目 (批准号: 2014RC02) 资助项目

摘要 $\bar{d}$ - 和 $\underline{d}$ - 跟踪性质是 Dastjerdi 和 Hosseini 为推广伪轨跟踪性质于 2010 年提出的. 本文考察该 动力性质在迭代系统和逆极限系统下的性质。首先证明对动力系统 $(X, f)$, 以下三命题等价: (1) $f$ 具 有 $\bar{d}$ - 跟踪性质 ( $\underline{d}$ - 跟踪性质); (2) 对任意 $k \in \mathbb{N}, f^{k}$ 也具有 $\bar{d}$ - 跟踪性质 ( $\underline{d}$ - 跟踪性质); (3) 存在 $k \in \mathbb{N}$, 使得 $f^{k}$ 具有 $\bar{d}$ - 跟踪性质 ( - 跟踪性质). 进而证明具有 $\bar{d}$ - 跟踪性质的系统是链混合的. 最后得到对 于由 $\left\{X_{i}, \varphi_{i}, f_{i}\right\}_{i=1}^{\infty}$ 生成的逆极限系统 $\left(X_{\infty}, f_{\infty}\right)$, 若每个 $f_{i}$ 均具有 $\bar{d}$ - 跟踪性质 (或者, $\underline{d}$ - 跟踪性质, 遍历跟踪性), 则诱导映射 $f_{\infty}$ 也具有 $\bar{d}$ - 跟踪性质 (相应地, $\underline{d}$ - 跟踪性质, 遍历跟踪性).

关键词 $\bar{d}$ - 跟踪性质 $\underline{d}$ - 跟踪性质 链混合 逆极限系统

MSC (2010) 主题分类 $54 \mathrm{H} 20,37 \mathrm{~B} 40,37 \mathrm{D} 45,28 \mathrm{D} 20$

\section{1 引言及基本定义}

设 $(X, d)$ 为紧致度量空间, 若映射 $f: X \longrightarrow X$ 为连续满射, 则称 $(X, f)$ 为一动力系统. 在动力系 统的研究中, 由于大部分系统的精确解无法得到, 学者们常用数值计算的方法来模拟所研究系统的轨 道, 而计算误差的存在便不可避免地导致 “伪轨” 的产生. 众所周知, 如果一个系统具有 “通常意义下 的伪轨跟踪性”, 则对任意一个单步误差足够小的伪轨必然被某条真实轨道跟踪, 并且其 “跟踪误差” 一致有界. 这说明在某种意义下, 数值计算得到的伪轨能够真实反映系统的局部动力学行为.

以下首先给出伪轨跟踪性的基本定义. 为后面叙述方便, 我们引入如下的记号. 贯穿全文, 置 $\mathbb{N}=$ $\{1,2,3, \ldots\}, \mathbb{Z}^{+}=\{0,1,2, \ldots\}$. 对于 $X$ 的任意一个无限序列 $\mathbb{x}:=\left\{x_{i}\right\}_{i=0}^{\infty}$ 和任意 $z \in X$, 任意 $\delta>0$, 令

$$
\begin{aligned}
& B_{n}(\mathbb{x}, f, \delta)=\left\{i \in \mathbb{Z}^{+}: 0 \leqslant i<n, d\left(f\left(x_{i}\right), x_{i+1}\right)<\delta\right\}, \\
& B_{n}^{c}(\mathbb{x}, f, \delta)=\left\{i \in \mathbb{Z}^{+}: 0 \leqslant i<n, d\left(f\left(x_{i}\right), x_{i+1}\right) \geqslant \delta\right\}, \\
& B_{n}(z, \mathbb{x}, f, \delta)=\left\{i \in \mathbb{Z}^{+}: 0 \leqslant i<n, d\left(f^{i}(z), x_{i}\right)<\delta\right\}, \\
& B_{n}^{c}(z, \mathbb{x}, f, \delta)=\left\{i \in \mathbb{Z}^{+}: 0 \leqslant i<n, d\left(f^{i}(z), x_{i}\right) \geqslant \delta\right\} .
\end{aligned}
$$

$X$ 中的序列 $\mathbb{x}=\left\{x_{i}\right\}_{i=0}^{K}$ 被称为是 $f$ 的 $\delta$-伪轨 ( $\delta$-pseudo-orbit), 如果对任意 $0 \leqslant i<K$, 恒有 $d\left(f\left(x_{i}\right), x_{i+1}\right)<\delta$. 特别地, 若 $K<\infty$, 则称这个有限的 $\delta$ - 伪轨 $\left\{x_{i}\right\}_{i=0}^{K}$ 为 $f$ 的从 $x_{0}$ 到 $x_{K}$ 的长度为 $K$ 的 $\delta$ - 链. 如果对任意 $\delta>0$ 及任意 $x, y \in X$, 均存在从 $x$ 到 $y$ 的 $\delta$ - 链, 则称 $f$ 是链传递的. 如果 
对任意 $n \in \mathbb{N}, f^{n}$ 均为链传递的, 则称 $f$ 是完全链传递的. 如果对任意 $\varepsilon>0$, 存在 $N \in \mathbb{N}$, 使得对任 意 $x, y \in X$ 及任意 $n \geqslant N, f$ 存在从 $x$ 到 $y$ 长度为 $n$ 的 $\varepsilon$ - 链, 则称 $f$ 是链混合的.

如果对任意 $\varepsilon>0$, 存在 $\delta>0$, 使得对 $f$ 的任意 $\delta$ - 伪轨 $\left\{x_{i}\right\}_{i=0}^{\infty}$, 存在 $z \in X$ 满足对任意 $i \in \mathbb{Z}^{+}$, $d\left(f^{i}(z), x_{i}\right)<\varepsilon$, 则称 $(X, f)$ 具有伪轨跟踪性质.

由伪轨跟踪性质的定义及现实意义下的动力系统数值计算方法知, 跟踪性质有两个基本要素: (1) 伪轨的刻画; (2) 跟踪方式的刻画. 基于对以上两个基本要素的不同理解, 学者们相继提出各种各样 的跟踪性质, 如平均跟踪性质 (average shadowing property) ${ }^{[1]}$, 极限跟踪性质 (limit shadowing property) ${ }^{[2]}$, 渐近平均跟踪性质 (asymptotic average shadowing property) ${ }^{[3]}$, 遍历跟踪性质 (ergodic shadowing property) ${ }^{[4]}, \bar{d}$-和 $\underline{d}$ - 跟踪性质 $\left(\bar{d}\right.$ - and $\underline{d}$-shadowing property) ${ }^{[5]}$ 以及 $\mathscr{F}$ - 跟踪性质 ( $\mathscr{F}$-shadowing property) ${ }^{[6]}$ 等. 在此, 我们仅给出本文需要的 “跟踪性” 的定义. 对于其他的跟踪性质, 请读者参见相 关文献 (如, 文献 [1-16]), 此处概不赘述.

定义 1.1 (参见文献 [1]) (1) $X$ 中的序列 $\mathbb{x}=\left\{x_{i}\right\}_{i=0}^{\infty}$ 被称为是 $f$ 的 $\delta$ - 平均伪轨 ( $\delta$-averagepseudo-orbit), 如果存在 $N_{\delta}>0$, 使得对任意 $n \geqslant N_{\delta}$ 及任意 $k \in \mathbb{Z}^{+}$, 恒有 $\frac{1}{n} \sum_{i=0}^{n-1} d\left(f\left(x_{i+k}\right), x_{i+k+1}\right)<$ $\delta$. 称系统 $(X, f)$ 具有平均跟踪性质, 如果对任意 $\varepsilon>0$, 存在 $\delta>0$, 使得对 $f$ 的任意 $\delta$ - 平均伪轨 $\left\{x_{i}\right\}_{i=0}^{\infty}$, 存在 $z \in X$ 满足 $\lim _{\sup } \rightarrow \infty \frac{1}{n} \sum_{i=0}^{n-1} d\left(f^{i}(z), x_{i}\right)<\varepsilon$.

(2) $X$ 中的序列 $\mathbb{X}=\left\{x_{i}\right\}_{i=0}^{\infty}$ 被称为是 $f$ 的渐近平均伪轨 (asymptotic-average pseudo-orbit), 如果 $\lim _{n \rightarrow \infty} \frac{1}{n} \sum_{i=0}^{n-1} d\left(f\left(x_{i}\right), x_{i+1}\right)=0$. 称系统 $(X, f)$ 具有渐近平均跟踪性质, 如果对 $f$ 的任意渐近平均 伪轨 $\left\{x_{i}\right\}_{i=0}^{\infty}$, 存在 $z \in X$, 使得 $\lim _{n \rightarrow \infty} \frac{1}{n} \sum_{i=0}^{n-1} d\left(f^{i}(z), x_{i}\right)=0$.

2010 年, Fakhari 和 Ghane ${ }^{[4]}$ 将 $\delta$ - 伪轨推广到 $\delta$ - 遍历伪轨 ( $\delta$-ergodic pseudo-orbit), 并提出了遍历 跟踪性质的概念. 利用遍历跟踪性质, 他们同时证明了以下 4 命题等价: (1) $f$ 具有遍历跟踪性质; (2) $f$ 具有伪轨跟踪性质和链混合的; (3) $f$ 具有伪轨跟踪性质和拓扑混合的; (4) $f$ 具有伪轨 specification 性质.

定义 1.2 (参见文献 [4]) 如果 $\lim _{n \rightarrow \infty} \frac{1}{n}\left|B_{n}(\mathbb{x}, f, \delta)\right|=1$, 则称 $X$ 中的序列 $\mathbb{x}=\left\{x_{i}\right\}_{i=0}^{\infty}$ 是 $f$ 的 $\delta$ - 遍历伪轨.

定义 1.3 (参见文献 [4]) 如果对任意 $\varepsilon>0$, 存在 $\delta>0$, 使得对 $f$ 的任意 $\delta$ - 遍历伪轨 $\mathbb{x}$, 存在 $z \in X$ 满足 $\lim _{n \rightarrow \infty} \frac{1}{n}\left|B_{n}(z, \mathbb{x}, f, \varepsilon)\right|=1$, 则称系统 $(X, f)$ 具有遍历跟踪性质.

几乎在同时, Dastjerdi 和 Hosseini ${ }^{[5]}$ 利用 $\delta$ - 遍历伪轨定义了 $\bar{d}$ - 跟踪性质和 $\underline{d}$ - 跟踪性质.

定义 1.4 (参见文献 [5, 定义 2.1]) 如果对任意 $\varepsilon>0$, 存在 $\delta>0$, 使得对 $f$ 的任意 $\delta$ - 遍历伪轨 $\mathrm{x}$, 存在 $z \in X$ 满足 $\limsup _{n \rightarrow \infty} \frac{1}{n}\left|B_{n}(z, \mathrm{x}, f, \varepsilon)\right|>1 / 2$ (或者, $\liminf _{n \rightarrow \infty} \frac{1}{n}\left|B_{n}(z, \mathbb{x}, f, \varepsilon)\right|>0$ ), 则称系 统 $(X, f)$ 具有 $\bar{d}$ - 跟踪性质 (相应地, $\underline{d}$ - 跟踪性质).

对于 $\underline{d}$ - 跟踪性质, Dastjerdi 和 Hosseini ${ }^{[5]}$ 证明了如下结论:

定理 1.5 (参见文献 [5, 定理 2.2 , 定理 2.5]) 假设 $(X, f)$ 为一动力系统.

(1) 若 $f$ 具有 $\underline{d}$ - 跟踪性质或者 $\bar{d}$ - 跟踪性质, 则 $f$ 是链传递的.

(2) 若 $f$ 具有 $\underline{d}$ - 跟踪性质, 则对任意 $k \in \mathbb{N}, f^{k}$ 均具有 $\underline{d}$ - 跟踪性质.

受此启发, 我们自然要问: 对于 $\bar{d}$ - 跟踪性质是否具有和定理 1.5 中 (1) 和 (2) 相似的结论, 同时, $\underline{d}$ 和 $\bar{d}$ - 跟踪性质在逆极限系统中是否保持呢?

我们发现, 文献 [5] 中虽然同时提出 $\bar{d}$ - 跟踪性质和 $\underline{d}$ - 跟踪性质, 却未能得出 $\bar{d}$ - 跟踪性质的相 关动力学性质, 究其原因在于应用文献 [5] 中的方法无法回答 $\bar{d}$ - 跟踪性质是否具有迭代不变性, 自 然导致相关的结论不能应用到 $\bar{d}$ - 跟踪性质上. 本文利用三角不等式和映射的一致连续性, 首先证明 $\bar{d}$ - 跟踪性质的迭代不变性. 由此得出 $\bar{d}$ - 跟踪性质蕴含链混合性. 同时, 还就 $\bar{d}$ - 跟踪性质中上极限 
$\limsup _{n \rightarrow \infty} \frac{1}{n}\left|B_{n}(z, \mathbb{x}, f, \varepsilon)\right|$ 的取值是大于 “ $1 / 2$ ”, 而不是 $[0,1)$ 之间的其他实数给出了解释, 指出该取 值恰到好处. 本文最后研究逆极限系统的 $\bar{d}$ - 跟踪性质， $\underline{d}$ - 跟踪性质和遍历跟踪性质.

\section{$2 \bar{d}$ - 跟踪性质的一些基本性质}

正如引言所述, 本节致力于考察 $k$ - 次迭代系统 $\left(X, f^{k}\right)$ 的 $\bar{d}$ - 跟踪性质 (引理 2.1 和 2.2). 利用该 结论, 进一步得出系统 $(X, f)$ 是链混合的, 如果 $f$ 具有 $\bar{d}$ - 跟踪性质.

对任意 $A \subset \mathbb{Z}^{+}$及任意 $k \in \mathbb{N}$, 置 $A+k=\{j+k: j \in A\} \cap \mathbb{Z}^{+}, A-k=\{j-k: j \in A\} \cap \mathbb{Z}^{+}$, $k \cdot A=\{k \cdot j: j \in A\}$. 对任意 $A_{1}, \ldots, A_{n} \subset \mathbb{Z}^{+}$, 不难证明对任意 $k \in \mathbb{N}$,

$$
\bigcap_{i=1}^{n}\left(A_{i}+k\right)=\left(\bigcap_{i=1}^{n} A_{i}\right)+k .
$$

引理 2.1 设 $(X, f)$ 为一动力系统. 如果 $f$ 具有 $\bar{d}$ - 跟踪性质, 则对任意 $k \in \mathbb{N}, f^{k}$ 具有 $\bar{d}$ - 跟踪 性质.

证明 任意给定 $\varepsilon>0$, 注意到 $f$ 一致连续, 则存在 $\varepsilon_{1} \in(0, \varepsilon / 4)$, 使得

$$
\text { 对任意 } x, y \in X \text { 满足 } d(x, y)<\varepsilon_{1} \text { 和任意 } 0 \leqslant i \leqslant k \text {, 恒有 } d\left(f^{i}(x), f^{i}(y)\right)<\frac{\varepsilon}{4} \text {. }
$$

由于 $f$ 具有 $\bar{d}$ - 跟踪性质, 所以存在 $\delta \in\left(0, \varepsilon_{1}\right)$, 使得对 $f$ 的任意 $\delta$ - 遍历伪轨 $\left\{y_{i}\right\}_{i=0}^{\infty}$, 存在 $y \in X$ 满足 $\limsup _{n \rightarrow \infty} \frac{1}{n}\left|B_{n}\left(y,\left\{y_{i}\right\}_{i=0}^{\infty}, f, \varepsilon_{1}\right)\right|>1 / 2$.

任取 $f^{k}$ 的 $\delta$ - 遍历伪轨 $\mathbb{x}=\left\{e_{i}\right\}_{i=0}^{\infty}$, 同时置 $x_{i k+j}=f^{j}\left(e_{i}\right)\left(\forall i \in \mathbb{Z}^{+}, 0 \leqslant j<k\right)$.

易知 $\mathrm{y}:=\left\{x_{i}\right\}_{i=0}^{\infty}$ 为 $f$ 的一个 $\delta$ - 遍历伪轨. 由以上的讨论知

$$
\text { 存在 } z \in X \text {, 使得 } \limsup _{n \rightarrow \infty} \frac{1}{n}\left|B_{n}\left(z, \mathbb{y}, f, \varepsilon_{1}\right)\right|>\frac{1}{2} \text {. }
$$

断言 $\limsup _{n \rightarrow \infty} \frac{1}{n}\left|B_{n}\left(z, \mathbb{x}, f^{k}, \varepsilon\right)\right|>1 / 2$.

断言的证明 (反证法) 假设 $\lim \sup _{n \rightarrow \infty} \frac{1}{n}\left|B_{n}\left(z, \mathbb{x}, f^{k}, \varepsilon\right)\right| \leqslant 1 / 2$, 由于

$$
\frac{1}{n}\left(\left|B_{n}\left(z, \mathbb{x}, f^{k}, \varepsilon\right)\right|+\left|B_{n}^{c}\left(z, \mathbb{x}, f^{k}, \varepsilon\right)\right|\right)=1,
$$

则有

$$
\xi:=\liminf _{n \rightarrow \infty} \frac{1}{n}\left|B_{n}^{c}\left(z, \mathbb{x}, f^{k}, \varepsilon\right)\right|=1-\limsup _{n \rightarrow \infty} \frac{1}{n}\left|B_{n}\left(z, \mathbb{x}, f^{k}, \varepsilon\right)\right| \geqslant \frac{1}{2} .
$$

所以对任意取定的 $Q \in \mathbb{N}$, 存在 $N_{Q}^{(1)} \in \mathbb{N}$, 使得任意 $n \geqslant N_{Q}^{(1)}$,

$$
\frac{1}{n}\left|B_{n}^{c}\left(z, \mathbb{x}, f^{k}, \varepsilon\right)\right| \geqslant \xi-\frac{1}{2 Q} .
$$

由于 $\lim _{n \rightarrow \infty} \frac{1}{n}\left|B_{n}\left(\mathbb{x}, f^{k}, \delta\right)+1\right|=1$, 同时结合

$$
\begin{aligned}
\left|\left(B_{n}\left(\mathbb{x}, f^{k}, \delta\right)+1\right) \cap B_{n}^{c}\left(z, \mathbb{x}, f^{k}, \varepsilon\right)\right|= & \left|\left(B_{n}\left(\mathbb{x}, f^{k}, \delta\right)+1\right)\right|+\left|B_{n}^{c}\left(z, \mathbb{x}, f^{k}, \varepsilon\right)\right| \\
& -\left|\left(B_{n}\left(\mathbb{x}, f^{k}, \delta\right)+1\right) \cup B_{n}^{c}\left(z, \mathbb{x}, f^{k}, \varepsilon\right)\right|,
\end{aligned}
$$

利用 (2.5) 式, 不难证明

$$
\liminf _{n \rightarrow \infty} \frac{1}{n}\left|\left(B_{n}\left(\mathbb{x}, f^{k}, \delta\right)+1\right) \cap B_{n}^{c}\left(z, \mathbb{x}, f^{k}, \varepsilon\right)\right| \geqslant \xi-\frac{1}{2 Q} .
$$


这便说明存在 $N_{Q}^{(2)} \in \mathbb{N}$, 使得任意 $n \geqslant N_{Q}^{(2)}$,

$$
\frac{1}{n}\left|\left(B_{n}\left(\mathbb{x}, f^{k}, \delta\right)+1\right) \cap B_{n}^{c}\left(z, \mathbb{x}, f^{k}, \varepsilon\right)\right| \geqslant \xi-\frac{1}{Q} .
$$

任取 $i \in \Omega_{n}:=\left(B_{n}\left(\mathbb{x}, f^{k}, \delta\right)+1\right) \cap B_{n}^{c}\left(z, \mathbb{x}, f^{k}, \varepsilon\right)\left(n \geqslant N_{Q}^{(2)}\right)$, 对任意 $1 \leqslant j<k$, 应用三角不等式

$$
\begin{aligned}
\varepsilon & \leqslant d\left(f^{i k}(z), x_{i k}\right) \leqslant d\left(f^{j}\left(f^{i k-j}(z)\right), f^{j}\left(x_{i k-j}\right)\right)+d\left(f^{j}\left(x_{i k-j}\right), x_{i k}\right) \\
& <d\left(f^{j}\left(f^{i k-j}(z)\right), f^{j}\left(x_{i k-j}\right)\right)+\delta\left(\text { 因 } f^{j}\left(x_{i k-j}\right)=f^{k}\left(x_{(i-1) k}\right) \text { 并且 } i-1 \in B_{n}\left(\mathbb{x}, f^{k}, \delta\right)\right),
\end{aligned}
$$

便有

$$
d\left(f^{j}\left(f^{i k-j}(z)\right), f^{j}\left(x_{i k-j}\right)\right)>\varepsilon-\delta>\frac{3 \varepsilon}{4} .
$$

由此和 $(2.2)$ 式可得对任意 $i \in \Omega_{n}\left(n \geqslant N_{Q}^{(2)}\right)$ 及任意 $1 \leqslant j<k$, 恒有

$$
d\left(f^{i k-j}(z), x_{i k-j}\right) \geqslant \varepsilon_{1}
$$

所以对任意 $n \geqslant N_{Q}^{(2)}$,

$$
B_{n k}^{c}\left(z, \mathbb{y}, f, \varepsilon_{1}\right) \supset \bigcup_{j=0}^{k-1}\left(k \cdot \Omega_{n}-j\right),
$$

注意到 $k \cdot \Omega_{n}, k \cdot \Omega_{n}-1, \ldots, k \cdot \Omega_{n}-(k-1)$ 是互不相交的, 进而

$$
\left|B_{n k}^{c}\left(z, \mathrm{y}, f, \varepsilon_{1}\right)\right| \geqslant\left|\bigcup_{j=0}^{k-1}\left(k \cdot \Omega_{n}-j\right)\right|=k\left|\Omega_{n}\right|,
$$

即

$$
\frac{\left|B_{n k}^{c}\left(z, \mathbb{y}, f, \varepsilon_{1}\right)\right|}{n k} \geqslant \frac{\left|\left(B_{n}\left(\mathbb{x}, f^{k}, \delta\right)+1\right) \cap B_{n}^{c}\left(z, \mathbb{x}, f^{k}, \varepsilon\right)\right|}{n} \geqslant \xi-\frac{1}{Q} .
$$

由此可知, 对任意 $m \geqslant N_{Q}^{(2)} \cdot k$, 恒有

$$
\begin{aligned}
\frac{\left|B_{m}^{c}\left(z, \mathbb{y}, f, \varepsilon_{1}\right)\right|}{m} & \geqslant \frac{\left|B_{[m / k]] k}^{c}\left(z, \mathbb{y}, f, \varepsilon_{1}\right)\right|}{([m / k]+1) k} \geqslant \frac{\left|B_{[m / k]+1) k}^{c}\left(z, \mathbb{y}, f, \varepsilon_{1}\right)\right|-k}{([m / k]+1) k} \\
& \geqslant \frac{\left|B_{[[m / k]+1) k}^{c}\left(z, \mathbb{y}, f, \varepsilon_{1}\right)\right|}{([m / k]+1) k}-\frac{1}{[m / k]+1} \geqslant \xi-\frac{1}{Q}-\frac{1}{[m / k]+1} .
\end{aligned}
$$

因此

$$
\liminf _{n \rightarrow \infty} \frac{1}{n}\left|B_{n}^{c}\left(z, \mathbb{y}, f, \varepsilon_{1}\right)\right| \geqslant \xi-\frac{1}{Q}
$$

由 $Q$ 的任意性, 故而

$$
\liminf _{n \rightarrow \infty} \frac{1}{n}\left|B_{n}^{c}\left(z, \mathbb{y}, f, \varepsilon_{1}\right)\right| \geqslant \xi \geqslant \frac{1}{2}
$$

由此不难证明

$$
\limsup _{n \rightarrow \infty} \frac{1}{n}\left|B_{n}\left(z, \mathbb{y}, f, \varepsilon_{1}\right)\right|=1-\liminf _{n \rightarrow \infty} \frac{1}{n}\left|B_{n}^{c}\left(z, \mathbb{y}, f, \varepsilon_{1}\right)\right| \leqslant 1-\xi \leqslant \frac{1}{2},
$$

这与 (2.3) 式矛盾, 断言得证.

由 $\varepsilon$ 及 $\mathbb{x}$ 的任意性自然得知, $f^{k}$ 具有 $\bar{d}$ - 跟踪性质. 
引理 2.2 设 $(X, f)$ 为一动力系统. 如果存在 $k \in \mathbb{N}$, 使得 $f^{k}$ 具有 $\bar{d}$ - 跟踪性质, 则 $f$ 具有 $\bar{d}$ - 跟 踪性质.

证明 任取 $\varepsilon>0$, 由文献 [17, 引理 1.2] 知, 存在 $\gamma \in(0, \varepsilon / 2)$, 使得对任意长度为 $k+1$ 的 $\gamma$ - 链 $\left\{x_{i}\right\}_{i=0}^{k+1}$ 及任意 $z \in \mathscr{N}\left(x_{0}, \gamma\right):=\left\{x \in X: d\left(x, x_{0}\right)<\gamma\right\}$, 恒有 $d\left(f^{i}(z), x_{i}\right)<\varepsilon(\forall i \in\{0,1, \ldots, k\})$.

由于 $f^{k}$ 具有 $\bar{d}$ - 跟踪性质, 则对于 $\gamma / 4>0$, 存在 $\delta \in(0, \gamma / 4)$, 使得对 $f^{k}$ 的任意 $\delta$ - 遍历为轨 $\left\{y_{i}\right\}_{i=0}^{\infty}$, 存在 $y \in X$ 满足 $\limsup _{n \rightarrow \infty} \frac{1}{n}\left|B_{n}\left(y,\left\{y_{i}\right\}_{i=0}^{\infty}, f^{k}, \gamma / 4\right)\right|>1 / 2$. 再次利用文献 [17, 引理 1.2], 存 在 $\delta^{\prime} \in(0, \delta / 2)$, 使得对任意长度为 $k+1$ 的 $\delta^{\prime}-$ 链 $\left\{x_{i}\right\}_{i=0}^{k+1}$ 及任意 $z \in \mathscr{N}\left(x_{0}, \delta^{\prime}\right)$, 恒有 $d\left(f^{i}(z), x_{i}\right)<\delta$ $(\forall i \in\{0,1, \ldots, k\})$.

任取 $f$ 的 $\delta^{\prime}$ - 遍历伪轨 $\mathbb{x}=\left\{x_{i}\right\}_{i=0}^{\infty}$, 同时置 $\mathbb{Z}=\left\{z_{i}=x_{i k}\right\}_{i=0}^{\infty}$, 并且

$$
\mathscr{C}_{n}:=\bigcap_{j=0}^{k}\left\{0 \leqslant i<n: d\left(f\left(x_{i k+j}\right), x_{i k+j+1}\right)<\delta^{\prime}\right\}, \quad \mathscr{C}=\bigcup_{n \in \mathbb{N}} \mathscr{C}_{n} .
$$

注意到 $\lim _{n \rightarrow \infty} \frac{1}{n}\left|B_{n}\left(\mathbb{x}, f, \delta^{\prime}\right)\right|=1$, 不难证明对于任意 $j \in\{0, \ldots, k\}$,

$$
\lim _{n \rightarrow \infty} \frac{1}{n}\left|\left\{0 \leqslant i<n: d\left(f\left(x_{i k+j}\right), x_{i k+j+1}\right)<\delta^{\prime}\right\}\right|=1 .
$$

因此, $\lim _{n \rightarrow \infty} \frac{1}{n}\left|\mathscr{C}_{n}\right|=1$. 任取 $i \in \mathscr{C}$, 则有对任意 $j \in\{0, \ldots, k\}, d\left(f\left(x_{i k+j}\right), x_{i k+j+1}\right)<\delta^{\prime}$. 由此结合 $\delta^{\prime}$ 的取法自有 $d\left(f^{k}\left(z_{i}\right), z_{i+1}\right)=d\left(f^{k}\left(x_{i k}\right), x_{i k+k}\right)<\delta$. 这说明 $\mathbb{Z}$ 为 $f^{k}$ 的一条 $\delta$ - 遍历伪轨. 因此

$$
\text { 存在 } z \in X \text {, 使得 } \limsup _{n \rightarrow \infty} \frac{1}{n}\left|B_{n}\left(z, \mathbb{Z}, f^{k}, \gamma / 4\right)\right|>1 / 2 \text {. }
$$

由此可得, 存在 $\xi>1 / 2$ 及严格递增序列 $\left\{n_{l}\right\}_{l=1}^{\infty} \subset \mathbb{N}$, 使得对任意 $l \in \mathbb{N}$, 恒有

$$
\frac{1}{n_{l}}\left|B_{n_{l}}\left(z, \mathbb{Z}, f^{k}, \gamma / 4\right)\right| \geqslant \xi .
$$

对任意 $j \in \mathscr{Q}_{n_{l}}:=\left[k \cdot B_{n_{l}}\left(z, \mathbb{Z}, f^{k}, \gamma / 4\right)\right] \cap\left(\bigcap_{i=0}^{k-1}\left(B\left(\mathbb{x}, f, \delta^{\prime}\right)-i\right)\right)$ (其中, $B\left(\mathbb{x}, f, \delta^{\prime}\right)=\bigcup_{n \in \mathbb{N}} B_{n}\left(\mathbb{x}, f, \delta^{\prime}\right)$ ), 注意到 $d\left(f\left(x_{j+i}\right), x_{j+i+1}\right)<\delta^{\prime}<\gamma(\forall i \in\{0,1, \ldots, k-1\})$ 并且 $d\left(f^{j}(z), x_{j}\right)=d\left(\left(f^{k}\right)^{j / k}(z), x_{(j / k) k}\right)<$ $\gamma / 4$ (因 $j \in k \cdot B_{n_{l}}\left(z, \mathbb{Z}, f^{k}, \gamma / 4\right)$ ), 由 $\gamma$ 的取法则有, 对任意 $i \in\{0,1, \ldots, k-1\}, d\left(f^{j+i}(z), x_{j+i}\right)<\varepsilon$. 由此可知,

$$
B_{\left(n_{l}+1\right) k}(z, \mathbb{x}, f, \varepsilon) \supset \bigcup_{i=0}^{k-1}\left(\mathscr{Q}_{n_{l}}+i\right) .
$$

注意到 $\lim _{n \rightarrow \infty} \frac{1}{n}\left|B_{n}\left(\mathrm{x}, f, \delta^{\prime}\right)\right|=1$, 不难证明

$$
\lim _{n \rightarrow \infty} \frac{1}{n}\left|[0, n) \cap\left(\bigcap_{i=0}^{k-1}\left(B\left(\mathbb{x}, f, \delta^{\prime}\right)-i\right)\right)\right|=1 .
$$

为证明方便, 记 $\mathscr{H}_{n}=[0, n) \cap\left(\bigcap_{i=0}^{k-1}\left(B\left(\mathbb{x}, f, \delta^{\prime}\right)-i\right)\right)$. 显然, 对任意 $0 \leqslant i<k, \mathscr{Q}_{n_{l}}+i \subset\left[0, n_{l} k+i\right)$, 所 以由 (2.1) 式有

$$
\mathscr{Q}_{n_{l}}+i=\left(\mathscr{Q}_{n_{l}}+i\right) \cap\left[0, n_{l} k+i\right)=\left(k \cdot B_{n_{l}}\left(z, \mathbb{Z}, f^{k}, \gamma / 4\right)+i\right) \cap\left(\mathscr{H}_{n_{l} k+i}+i\right),
$$

故而

$$
\begin{aligned}
\left|\left(\mathscr{Q}_{n_{l}}+i\right)\right|= & \left|\left(k \cdot B_{n_{l}}\left(z, \mathbb{Z}, f^{k}, \gamma / 4\right)+i\right)\right|+\left|\left(\mathscr{H}_{n_{l} k+i}+i\right)\right| \\
& -\left|\left(k \cdot B_{n_{l}}\left(z, \mathbb{Z}, f^{k}, \gamma / 4\right)+i\right) \cup\left(\mathscr{H}_{n_{l} k+i}+i\right)\right| .
\end{aligned}
$$


由此和 $(2.14)$ 及 $(2.16)$ 式, 我们有

$$
\begin{aligned}
\liminf _{l \rightarrow \infty} \frac{1}{\left(n_{l}+1\right) k}\left|\left(\mathscr{Q}_{n_{l}}+i\right)\right| & =\liminf _{l \rightarrow \infty} \frac{1}{\left(n_{l}+1\right) k}\left|\left(k \cdot B_{n_{l}}\left(z, \mathbb{Z}, f^{k}, \gamma / 4\right)+i\right)\right| \\
& \geqslant \liminf _{l \rightarrow \infty} \frac{n_{l} \xi}{\left(n_{l}+1\right) k}=\frac{\xi}{k} .
\end{aligned}
$$

注意到 $\mathscr{Q}_{n_{l}}, \mathscr{Q}_{n_{l}}+1, \ldots, \mathscr{Q}_{n_{l}}+k-1$ 是互不相交的, 则

$$
\begin{aligned}
\limsup _{n \rightarrow \infty} \frac{1}{n}\left|B_{n}(z, \mathbb{x}, f, \varepsilon)\right| & \geqslant \liminf _{l \rightarrow \infty} \frac{1}{\left(n_{l}+1\right) k}\left|B_{\left(n_{l}+1\right) k}(z, \mathbb{x}, f, \varepsilon)\right| \\
& \geqslant \liminf _{l \rightarrow \infty} \frac{1}{\left(n_{l}+1\right) k} \sum_{i=0}^{k-1}\left|\left(\mathscr{Q}_{n_{l}}+i\right)\right| \text { (由 (2.15) 式) } \\
& \geqslant \sum_{i=0}^{k-1} \liminf _{l \rightarrow \infty} \frac{1}{\left(n_{l}+1\right) k}\left|\left(\mathscr{Q}_{n_{l}}+i\right)\right| \geqslant \xi>\frac{1}{2} .
\end{aligned}
$$

由 $\varepsilon$ 及 $\mathbb{x}$ 的任意性自然可得, $f$ 具有 $\bar{d}$ - 跟踪性质.

由引理 2.2 的证明过程, 不难发现对任意 $\delta>0$ 及任意 $k \in \mathbb{N}$, 存在 $\delta^{\prime}>0$, 使得对 $f$ 的任意 $\delta^{\prime}$ 遍历伪轨 $\left\{x_{i}\right\}_{i=0}^{\infty}$, 恒有 $\left\{x_{i k}\right\}_{i=0}^{\infty}$ 为 $f^{k}$ 的 $\delta$ - 遍历伪轨. 同时, 不难证明: 对任意 $z \in X$ 和任意 $\varepsilon>0$,

$$
\begin{aligned}
\liminf _{n \rightarrow \infty} \frac{1}{n}\left|B_{n}\left(z,\left\{x_{i}\right\}_{i=0}^{\infty}, f, \varepsilon\right)\right| & =\liminf _{n \rightarrow \infty} \frac{1}{n k}\left|B_{n k}\left(z,\left\{x_{i}\right\}_{i=0}^{\infty}, f, \varepsilon\right)\right| \\
& \geqslant \liminf _{n \rightarrow \infty} \frac{1}{n k}\left|B_{n}\left(z,\left\{x_{i k}\right\}_{i=0}^{\infty}, f^{k}, \varepsilon\right)\right| .
\end{aligned}
$$

由此, 我们便有以下结论成立.

引理 2.3 设 $(X, f)$ 为一动力系统. 如果存在 $k \in \mathbb{N}$, 使得 $f^{k}$ 具有 $\underline{d}$ - 跟踪性质, 则 $f$ 具有 $\underline{d}$ - 跟 踪性质.

综合引理 2.1-2.3 及文献 [5, 定理 2.5], 自然可得以下命题成立.

定理 2.4 设 $(X, f)$ 为一动力系统, 则以下命题等价:

(1) $f$ 具有 $\bar{d}$ - 跟踪性质 ( $\underline{d}$ - 跟踪性质);

(2) 存在 $k \in \mathbb{N}$, 使得 $f^{k}$ 具有 $\bar{d}$ - 跟踪性质 ( $\underline{d}$ - 跟踪性质);

(3) 对任意 $k \in \mathbb{N}, f^{k}$ 具有 $\bar{d}$ - 跟踪性质 ( $\underline{d}$ - 跟踪性质).

定理 2.5 如果系统 $(X, f)$ 具有 $\bar{d}$ - 跟踪性质, 则 $f$ 是链混合的.

证明 由文献 [15, 推论 12] 知: 完全链传递等价于链混合. 应用引理 2.1 和文献 [5, 定理 2.2], 我 们不难得出 $f$ 是完全链传递的, 该命题得证.

定理 2.6 动力系统 $(X, f)$ 具有 $\bar{d}$ - 跟踪性质 (或者, $\underline{d}$ - 跟踪性质) 当且仅当 $(X, f)$ 是链混合的, 并且满足对任意 $\varepsilon>0$, 存在 $\delta>0$, 使得对 $f$ 的任意 $\delta$ - 伪轨 $\mathbb{x}$, 存在 $z \in X$ 满足

$$
\limsup _{n \rightarrow \infty} \frac{1}{n}\left|B_{n}(z, \mathbf{x}, f, \varepsilon)\right|>1 / 2
$$

相应地, $\liminf _{n \rightarrow \infty} \frac{1}{n}\left|B_{n}(z, \mathbb{x}, f, \varepsilon)\right|>0$.

证明 关于 $\underline{d}$ - 跟踪性质可以由文献 [5, 引理 4.1] 直接得到; 应用定理 2.5 , 类似于文献 [5, 引 理 4.1] 的证明, 不难得出该命题对 $\bar{d}$ - 跟踪性质也成立.

如果对 $X$ 的任意两个非空开子集 $U_{0}, U_{1}$, 总存在 $N \in \mathbb{N}$, 使得对任意 $k \geqslant 2$ 及任意 $S=$ $\{s(1), \ldots, s(k)\} \in\{0,1\}^{k}$, 存在 $x \in X$ 满足 $x \in U_{s(1)}$ 并且 $f^{N}(x) \in U_{s(2)}, \ldots, f^{(k-1) N}(x) \in U_{s(k)}$, 
则称 $f$ 具有性质 $P$. 如果任意两个非稠密的开集构成 $X$ 的覆盖总有正拓扑熵, 则称 $f$ 有一致正熵. 如果 $(X, f)$ 的任何非平凡因子总有正拓扑熵, 则称 $f$ 有完全正熵.

如果对 $X$ 的任意两个非空开子集 $U, V$, 及任意 $\varepsilon>0$, 存在 $x \in U, y \in V$ 及 $n \in \mathbb{Z}^{+}$, 使得 $d\left(f^{n}(x), f^{n}(y)\right) \leqslant \varepsilon$, 则称 $f$ 是可达的. 如果 $f$ 是拓扑传递, 初值敏感依赖并且可达的, 则称 $f$ 是 Ruelle-Takens-Kato 意义下的混沌.

推论 2.7 如果系统 $(X, f)$ 具有伪轨跟踪性质, 则以下命题等价: (1) $f$ 具有遍历跟踪性质; $(2) f$ 是链混合的; (3) $f$ 具有 $\underline{d}$ - 跟踪性质; (4) $f$ 具有 $\bar{d}$ - 跟踪性质; (5) $f$ 是完全传递的; (6) $f$ 是拓扑混合 的; (7) $f$ 具有平均跟踪性质; (8) $f$ 具有 specification 性质; (9) $f$ 是拓扑弱混合的; (10) $f$ 有性质 $P$; (11) $f$ 有完全正熵; (12) $f$ 有一致正熵; (13) $f$ 是 Ruelle-Takens-Kato 意义下的混沌; (14) $f$ 是拓扑传 递且可达的.

证明 由文献 [4, 定理 A] 知, (1) (7), 同时, 应用定理 2.6, 并且由文献 [6, 引理 4 和定理 5] 证明过程易知, (7) $\Longrightarrow(4)$; 由文献 [14, 定 理 3] 知, $(2) \Longleftrightarrow(9) \Longleftrightarrow(10) \Longleftrightarrow(11) \Longleftrightarrow(12) \Longleftrightarrow(13) \Longleftrightarrow(14)$; 最后由定理 2.6 知, (4) $\Longrightarrow(2)$. 至此, 该命题得证.

注 2.8 (1) 由于一个子转移具有伪轨跟踪性质当且仅当它是有限型的, 所以推论 2.7 对于有限 型的子转移也是成立的. 由定理 2.6 和文献 [18, 定理 3.7], 类似于文献 [6, 定理 4] 的证明得知:

$$
\text { 渐近平均跟踪性质 } \Longrightarrow \text { 平均跟踪性质 } \Longrightarrow \bar{d} \text { - 跟踪性质 }+\underline{d} \text { - 跟踪性质. }
$$

由此结合定理 2.5 和推论 2.7 得 [3, 定理 3.1, 定理 4.1] 是显然成立的.

(2) 由文献 $\left[19\right.$, 定理 2.3.2] 知, 系统 $\left(X, \operatorname{id}_{X}\right)$ 具有伪轨跟踪性质当且仅当 $X$ 是完全不连通的. 然 而, 应用推论 2.7 易证满足该条件系统既不具有 $\bar{d}$ - 跟踪性质也不具有 $\underline{d}$ - 跟踪性质.

例 2.9 (参见文献 [20, 例 3.13]) 设 $\mathbb{S}^{1}$ 为单位圆周, 并且定义映射 $f$ 如下:

$$
f: \mathbb{S}^{1} \ni \mathrm{e}^{2 \pi \mathrm{i} x} \longmapsto \mathrm{e}^{2 \pi \mathrm{i} x^{2}} \in \mathbb{S}^{1}, \quad x \in[0,1] .
$$

文献 [20] 已证明 $f$ 具有渐近平均跟踪性质但不具有伪轨跟踪性质. 由此和 (2.19) 式及注 2.8 的 (2) 自 然可得 $\bar{d}$ 和 $\underline{d}$ - 跟踪性质与伪轨跟踪性质是互不蕴含的.

命题 2.10 不存在具有渐近平均跟踪性质的非平凡等度连续系统.

证明 由文献 [21, 定理 3.1] 知, 具有渐近平均跟踪性质的等度连续系统是拓扑传递的. 由此结 合文献 [22, 定理 4] 得, 这样的系统为极小系统. 而由文献 [5, 定理 2.8] 有, 该系统是拓扑弱混合的, 自 然初值敏感依赖的, 这与等度连续矛盾.

注 2.11 推论 2.10 表明满足文献 [21, 定理 3.1] 假设条件的系统是不存在的. 最近, 我们得到更 一般的结论: 不存在具有 $\underline{d}$ - 跟踪性质的非平凡等度连续系统.

虽然文献 [5] 早在 2010 年便给出了 $\bar{d}$ - 跟踪性质的确切定义, 然而让学者们倍感疑惑的是为什么 在定义 1.4 中上极限 $\limsup _{n \rightarrow \infty} \frac{1}{n}\left|B_{n}(z, \mathbb{x}, f, \varepsilon)\right|$ 的取值是大于 “ $1 / 2$ ”, 而不是 $[0,1)$ 之间的其他实数. 对于该问题, 文献 [5] 的作者们并未给出具体的说明. 作为本节结尾, 我们将从 “链传递性” 的角度说 明取值 $1 / 2$ 恰到好处. 为此, 先引入如下一些基本定义:

如果对任意 $\varepsilon>0$, 存在 $\delta>0$, 使得对 $f$ 的任意 $\delta$ - 遍历伪轨 $\mathrm{x}$, 存在 $z \in X$ 满足

$$
\limsup _{n \rightarrow \infty} \frac{1}{n}\left|B_{n}(z, \mathbb{x}, f, \varepsilon)\right|>\alpha\left(\text { 或者, } \liminf _{n \rightarrow \infty} \frac{1}{n}\left|B_{n}(z, \mathbb{x}, f, \varepsilon)\right|>\alpha\right),
$$


则称系统 $(X, f)$ 具有 $\mathscr{M}^{\alpha}$ - 跟踪性质 (相应地, $\mathscr{M}_{\alpha^{-}}$跟踪性质), 其中 $\alpha \in[0,1)$.

显然 $\mathscr{M}^{1 / 2}$ - 跟踪性质和 $\mathscr{M}_{0}$ - 跟踪性质分别就是定义 1.4 中的 $\bar{d}$ - 跟踪性质和 $\underline{d}$ - 跟踪性质. 因 此, $\left\{\alpha \in[0,1): \mathscr{M}_{\alpha^{-}}\right.$跟踪性质 $\Longrightarrow$ 链传递性 $\}=[0,1)$. 应用定理 2.5 , 自然有对任意 $\alpha \in[1 / 2,1)$, 具有 $\mathscr{M}^{\alpha}$ - 跟踪性质的系统是链传递, 甚至链混合的. 下面的例子表明对任意 $\alpha \in[0,1 / 2)$, 存在具有 $\mathscr{M}^{\alpha}-$ 跟踪性质却不是链传递的系统.

例 2.12 取 $X_{0}=[0,1], X_{1}=[2,3]$, 同时定义映射 $g: X_{0} \cup X_{1} \longrightarrow X_{0} \cup X_{1}$ 为

$$
g(x)= \begin{cases}1-|1-2 x|, & x \in X_{0}, \\ 3-|1-2(x-2)|, & x \in X_{1} .\end{cases}
$$

显然 $g$ 不是链传递的, 因为 $X_{0}, X_{1}$ 都是 $g$ 的闭不变子集. 因此, $g$ 不具有 $\bar{d}$ - 跟踪性质.

由文献 [4, 例] 得子系统 $\left.g\right|_{X_{0}}$ 及 $\left.g\right|_{X_{1}}$ 均具有遍历跟踪性质, 所以任意给定的 $\varepsilon \in(0,1)$, 存 在 $\delta>0$, 使得对 $\left.g\right|_{X_{0}}$ (或者, $\left.g\right|_{X_{1}}$ ) 的任意 $\delta$ - 遍历伪轨 $\mathbb{x}$, 存在 $z \in X_{0}$ (相应地, $z \in X_{1}$ ) 满足 $\lim _{n \rightarrow \infty} \frac{1}{n}\left|B_{n}\left(z, \mathbb{x},\left.g\right|_{X_{0}}, \varepsilon\right)\right|=1$ (相应地, $\lim _{n \rightarrow \infty} \frac{1}{n}\left|B_{n}\left(z, \mathbb{x},\left.g\right|_{X_{1}}, \varepsilon\right)\right|=1$ ).

任意取定 $g$ 的 $\delta$ - 遍历伪轨 $\left\{x_{i}\right\}_{i=0}^{\infty}$, 下证存在 $z \in X$, 使得 $\lim _{\sup } \sup _{n \rightarrow \infty} \frac{1}{n} B_{n}\left(z,\left\{x_{i}\right\}_{i=0}^{\infty}, g, \varepsilon\right) \geqslant 1 / 2$. 为此, 令

$$
\begin{aligned}
& \Gamma_{0}:=\left\{j \in \mathbb{Z}^{+}: x_{j} \in X_{1}, \text { 并且 } x_{j+1} \in X_{0}\right\}, \\
& \Gamma_{1}:=\left\{j \in \mathbb{Z}^{+}: x_{j} \in X_{0}, \text { 并且 } x_{j+1} \in X_{1}\right\} .
\end{aligned}
$$

不失一般性, 记 $\Gamma_{0}=\left\{n_{1}^{(0)}, n_{2}^{(0)}, n_{3}^{(0)}, \ldots\right\}, \Gamma_{1}=\left\{n_{1}^{(1)}, n_{2}^{(1)}, n_{3}^{(1)}, \ldots\right\}$, 同时可假定 $n_{1}^{(0)}<n_{1}^{(1)}<n_{2}^{(0)}<$ $n_{2}^{(1)}<\cdots$. 置 $\Pi_{0}:=\bigcup_{k=1}^{\infty}\left[n_{k}^{(0)}+1, n_{k}^{(1)}\right], \Pi_{1}=\left[0, n_{1}^{(0)}\right] \cup\left(\bigcup_{k=1}^{\infty}\left[n_{k}^{(1)}+1, n_{k+1}^{(0)}\right]\right)$. 不难验证, 对任意 $i \in \Pi_{j}(j \in\{0,1\}), x_{i} \in X_{j}$.

取

$$
x_{i}^{(0)}= \begin{cases}x_{i}, & i \in \Pi_{0}, \\ g^{i-\left(n_{1}^{(0)}+1\right)}\left(x_{n_{1}^{(0)}+1}\right), & i \in\left[0, n_{1}^{(0)}\right], \\ g^{i-n_{k}^{(1)}\left(x_{n_{k}^{(1)}}\right),} & i \in\left[n_{k}^{(1)}+1, n_{k+1}^{(0)}\right], k=1,2,3, \ldots,\end{cases}
$$

并且

$$
x_{i}^{(1)}= \begin{cases}x_{i}, & i \in \Pi_{1}, \\ g^{i-n_{k}^{(0)}}\left(x_{n_{k}^{(0)}}\right), & i \in\left[n_{k}^{(0)}+1, n_{k}^{(1)}\right], k=1,2,3, \ldots,\end{cases}
$$

易证 $\left\{x_{i}^{(0)}\right\}_{i=0}^{\infty}$ 和 $\left\{x_{i}^{(1)}\right\}_{i=0}^{\infty}$ 分别为子系统 $\left.g\right|_{X_{0}}$ 和 $\left.g\right|_{X_{1}}$ 的 $\delta$ - 遍历伪轨. 所以

$$
\text { 存在 } z^{j} \in X_{j}(j \in\{0,1\}) \text {, 使得 } \lim _{n \rightarrow \infty} \frac{1}{n}\left|B_{n}\left(z^{(j)},\left\{x_{i}^{(j)}\right\}_{i=0}^{\infty},\left.g\right|_{X_{j}}, \varepsilon\right)\right|=1 \text {. }
$$

记 $\Pi_{j}^{(n)}=\Pi_{j} \cap\{0,1, \ldots, n-1\}$. 结合

$$
\begin{aligned}
\left|B_{n}\left(z^{(j)},\left\{x_{i}^{(j)}\right\}_{i=0}^{\infty},\left.g\right|_{X_{j}}, \varepsilon\right) \cap \Pi_{j}^{(n)}\right|= & \left|B_{n}\left(z^{(j)},\left\{x_{i}^{(j)}\right\}_{i=0}^{\infty},\left.g\right|_{X_{j}}, \varepsilon\right)\right| \\
& +\left|\Pi_{j}^{(n)}\right|-\left|B_{n}\left(z^{(j)},\left\{x_{i}^{(j)}\right\}_{i=0}^{\infty},\left.g\right|_{X_{j}}, \varepsilon\right) \cup \Pi_{j}^{(n)}\right|,
\end{aligned}
$$

应用 $(2.20)$ 式则有

$$
\limsup _{n \rightarrow \infty} \frac{1}{n}\left|B_{n}\left(z^{(j)},\left\{x_{i}^{(j)}\right\}_{i=0}^{\infty},\left.g\right|_{X_{j}}, \varepsilon\right) \cap \Pi_{j}^{(n)}\right|=\limsup _{n \rightarrow \infty} \frac{1}{n}\left|\Pi_{j}^{(n)}\right| .
$$


进而, 不难证明对任意 $j \in\{0,1\}$,

$$
B_{n}\left(z^{(j)},\left\{x_{i}^{(j)}\right\}_{i=0}^{\infty},\left.g\right|_{X_{j}}, \varepsilon\right) \cap \Pi_{j}^{(n)}=B_{n}\left(z^{(j)},\left\{x_{i}\right\}_{i=0}^{\infty}, g, \varepsilon\right) .
$$

注意到 $\Pi_{0} \cup \Pi_{1}=\mathbb{Z}^{+}$, 因此存在 $j_{0} \in\{0,1\}$, 使得 $\lim _{\sup } \sup _{n \rightarrow \infty} \frac{1}{n}\left|\Pi_{j_{0}}^{(n)}\right| \geqslant 1 / 2$. 由此结合 (2.21)、(2.22) 式自有

$$
\limsup _{n \rightarrow \infty} \frac{1}{n} B_{n}\left(z^{\left(j_{0}\right)},\left\{x_{i}\right\}_{i=0}^{\infty}, g, \varepsilon\right)=\limsup _{n \rightarrow \infty} \frac{1}{n}\left|B_{n}\left(z^{\left(j_{0}\right)},\left\{x_{i}^{\left(j_{0}\right)}\right\}_{i=0}^{\infty},\left.g\right|_{X_{j_{0}}}, \varepsilon\right) \cap \Pi_{j_{0}}^{(n)}\right| \geqslant \frac{1}{2} .
$$

由 $\varepsilon$ 的任意性, 故而对任意 $\alpha \in[0,1 / 2)$, 系统 $(X, g)$ 具有 $\mathscr{M}^{\alpha}$ - 跟踪性质. 综合以上讨论则有

$$
\left\{\alpha \in[0,1): \mathscr{M}^{\alpha} \text { - 跟踪性质 } \Longrightarrow \text { 链传递性 }\right\}=[1 / 2,1) \text {. }
$$

这便意味着在保证链传递性意义下, $\bar{d}$ - 跟踪性质定义中 “ $1 / 2$ ” 的取值是恰到好处的.

类似于定理 2.4, 不难得到 $\mathscr{M}_{\alpha}$ 和 $\mathscr{M}^{\alpha}$ - 跟踪性质的迭代不变性.

定理 $\mathbf{2 . 1 3}$ 设 $(X, f)$ 为一动力系统, 并且 $\alpha \in[0,1)$, 则以下命题等价:

(1) $f$ 具有 $\mathscr{M}_{\alpha^{-}}$跟踪性质 $\left(\mathscr{M}^{\alpha}\right.$ - 跟踪性质);

(2) 存在 $k \in \mathbb{N}$, 使得 $f^{k}$ 具有 $\mathscr{M}_{\alpha^{-}}$跟踪性质 ( $\mathscr{M}^{\alpha}$ - 跟踪性质);

(3) 对任意 $k \in \mathbb{N}, f^{k}$ 具有 $\mathscr{M}_{\alpha^{-}}$跟踪性质 ( $\mathscr{M}^{\alpha}$ - 跟踪性质).

\section{3 逆极限系统的遍历伪轨跟踪性质}

设 $\left\{\left(X_{i}, d_{i}\right)\right\}_{i=1}^{\infty}$ 为一族紧致度量空间, 映射 $\varphi_{i}: X_{i+1} \longrightarrow X_{i}$ 为连续满射. 记

$$
X_{\infty}:=\lim _{\longleftarrow}\left\{X_{i}, \varphi_{i}\right\}=\left\{\left(x_{1}, x_{2}, x_{3}, \ldots\right) \in \prod_{i=1}^{\infty} X_{i}: \varphi_{i}\left(x_{i+1}\right)=x_{i}, \forall i \in \mathbb{N}\right\},
$$

并称 $X_{\infty}$ 为由 $\left\{X_{i}, \varphi_{i}\right\}_{i=1}^{\infty}$ 生成的逆极限空间, $\varphi_{i}$ 称为底映射. 容易验证, $X_{\infty}$ 为 $\prod_{i=1}^{\infty} X_{i}$ 的紧子空 间. 赋予 $X_{\infty}$ 如下相容度量:

$$
d_{\infty}(x, y)=\sum_{i=1}^{\infty} \frac{1}{2^{i}} \frac{d_{i}\left(x_{i}, y_{i}\right)}{1+d_{i}\left(x_{i}, y_{i}\right)}, \quad \forall x=\left(x_{1}, x_{2}, x_{3}, \ldots\right), y=\left(y_{1}, y_{2}, y_{3}, \ldots\right) \in X_{\infty} .
$$

若 $f_{i}: X_{i} \longrightarrow X_{i}$ 为连续映射并且满足 $\varphi_{i} \circ f_{i+1}=f_{i} \circ \varphi_{i}, \forall i \in \mathbb{N}$, 则 $\left\{f_{i}\right\}_{i=1}^{\infty}$ 在 $X_{\infty}$ 上诱导一个连续 映射

$$
\begin{aligned}
& f_{\infty}: X_{\infty} \longrightarrow X_{\infty} \\
& \left(x_{1}, x_{2}, x_{3}, \ldots\right) \longmapsto\left(f_{1}\left(x_{1}\right), f_{2}\left(x_{2}\right), f_{3}\left(x_{3}\right), \ldots\right) .
\end{aligned}
$$

称 $\left(X_{\infty}, f_{\infty}\right)$ 为由 $\left\{X_{i}, \varphi_{i}, f_{i}\right\}_{i=1}^{\infty}$ 生成的逆极限系统. 表示如下:

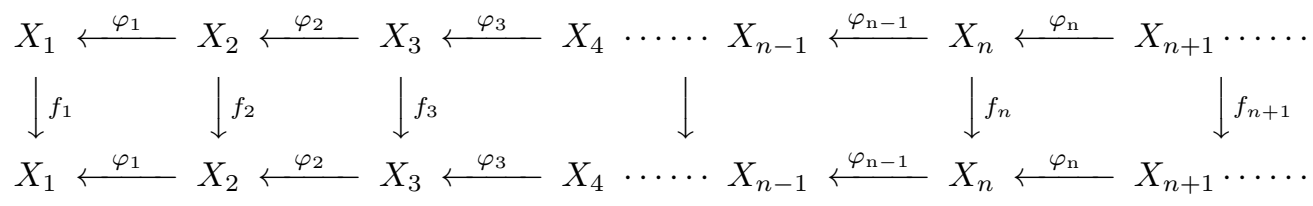


特别地, 如果对任意 $i \in \mathbb{N}, X_{i}=X, f_{i}=\varphi_{i}=f$, 则称

$$
X_{f}=\left\{\left(x_{1}, x_{2}, x_{3}, \ldots\right): f\left(x_{i+1}\right)=x_{i}, \forall i \in \mathbb{N}\right\},
$$

为 $f$ 的极限空间, $f$ 在 $X_{f}$ 上诱导一个同胚映射 $\sigma_{f}=f_{\infty}$, 称其为移位同胚. 对 $j \geqslant i$, 定义 $\varphi_{j, i}$ : $X_{j} \longrightarrow X_{i}$ 为 $\varphi_{j, i}=$ id 若 $j=i$; 而 $\varphi_{j, i}=\varphi_{i} \circ \varphi_{i+1} \circ \cdots \circ \varphi_{j-1}$ 若 $j>i$. 设 $X_{\infty}$ 为 $\left\{\left(X_{i}, \varphi_{i}\right)\right\}_{i=1}^{\infty}$ 生成 的逆极限空间, 定义投影映射 $\pi_{i}: X_{\infty} \longrightarrow X_{i}(i \in \mathbb{N})$ 为 $\pi_{i}\left(x_{1}, x_{2}, x_{3}, \ldots\right)=x_{i}$. 显然, $\pi_{i}$ 为连续满射 并且满足 $f_{i} \circ \pi_{i}=\pi_{i} \circ f_{\infty}$.

\section{1 逆极限系统 $\left(X_{\infty}, f_{\infty}\right)$ 的遍历伪轨跟踪性}

2001 年, 李思敏在文献 [23] 中证明了如果每个 $f_{i}$ 具有伪轨跟踪性质, 则诱导映射 $f_{\infty}$ 具有伪轨 跟踪性质. 顾荣宝 ${ }^{[24]}$ 和牛应轩 [25] 分别于 2006 和 2007 年证明了该命题对于渐近伪轨跟踪性质和渐 近平均伪轨跟踪性质都是成立的.

本小节致力于研究系统 $\left\{\left(X_{i}, f_{i}\right)\right\}_{i=1}^{\infty}$ 如何影响其生成的逆极限系统 $\left(X_{\infty}, f_{\infty}\right)$ 的遍历伪轨跟踪性 质. 对于此, 我们有如下定理:

定理 3.1 设 $\left(X_{\infty}, f_{\infty}\right)$ 为由 $\left\{\left(X_{i}, \varphi_{i}, f_{i}\right)\right\}_{i=1}^{\infty}$ 生成的逆极限系统. 如果每个 $f_{i}$ 具有 $\bar{d}$ - 跟踪性质, 则 $f_{\infty}$ 具有 $\bar{d}$ - 跟踪性质.

证明 任意给定 $\varepsilon>0$, 取定 $N \geqslant 2$ 满足 $\sum_{n=N+1}^{\infty} 1 / 2^{n}<\varepsilon / 2$. 由于任意 $1 \leqslant i \leqslant N, \varphi_{(N+1), i}$ 一 致连续, 所以存在 $\delta_{1} \in(0, \varepsilon / 2)$, 使得任意 $x, y \in X_{N+1}$ 满足 $d_{N+1}(x, y)<\delta_{1}$ 及任意 $1 \leqslant i \leqslant N$, 恒有

$$
d_{i}\left(\varphi_{(N+1), i}(x), \varphi_{(N+1), i}(y)\right)<\frac{\varepsilon}{2} .
$$

注意到 $f_{N+1}$ 具有 $\bar{d}$ - 跟踪性质, 所以存在 $\delta_{2} \in\left(0, \delta_{1}\right]$, 满足

$$
\text { 对 } f_{N+1} \text { 的任意 } \delta_{2^{-}} \text {遍历伪轨 } \mathrm{x} \text {, 存在 } z \in X_{N+1} \text {, 使得 } \limsup _{n \rightarrow \infty} \frac{1}{n}\left|B_{n}\left(z, \mathrm{x}, f_{N+1}, \delta_{1}\right)\right|>\frac{1}{2} \text {. }
$$

令 $\delta=\delta_{2} / 2^{N+1}\left(1+\delta_{2}\right)$. 任取 $f_{\infty}$ 的 $\delta$ - 遍历伪轨 $\mathbb{Y}=\left\{\mathrm{y}_{i}:=\left(y_{1}^{(i)}, y_{2}^{(i)}, y_{3}^{(i)}, \ldots\right)\right\}_{i=0}^{\infty} \subset X_{\infty}$, 同时 令 $\mathbb{Z}_{n}=\left\{y_{n}^{(i)}\right\}_{i=0}^{\infty}$. 由 $\delta$ 的选取, 容易验证 $\mathbb{Z}_{N+1}$ 为 $f_{N+1}$ 的一个 $\delta_{2}$ - 遍历伪轨. 由 (3.2) 式知, 存在 $\xi_{N+1} \in X_{N+1}$, 使得

$$
\limsup _{n \rightarrow \infty} \frac{1}{n}\left|B_{n}\left(\xi_{N+1}, \mathbb{Z}_{N+1}, f_{N+1}, \delta_{1}\right)\right|>\frac{1}{2} .
$$

由于每个 $\varphi_{i}$ 为满射, 我们可取 $\xi \in X_{\infty}$ 满足 $\pi_{N+1}(\xi)=\xi_{N+1}$. 不失一般性, 令 $\xi=\left(\xi_{1}, \xi_{2}, \ldots, \xi_{N+1}, \ldots\right)$. 不难证明: 对任意 $k \in \mathbb{Z}^{+}$及任意 $1 \leqslant i \leqslant N$,

$$
f_{i}^{k}\left(\xi_{i}\right)=f_{i}^{k} \circ \varphi_{(N+1), i}\left(\xi_{N+1}\right)=\varphi_{(N+1), i} \circ f_{N+1}^{k}\left(\xi_{N+1}\right) .
$$

由此和 $\delta_{1}$ 的选取及 $(3.1)$ 式可知, 对任意 $k \in \bigcup_{n \in \mathbb{N}} B_{n}\left(\xi_{N+1}, \mathbb{Z}_{N+1}, f_{N+1}, \delta_{1}\right)$ 及任意 $1 \leqslant i \leqslant N+1$,

$$
d_{i}\left(f_{i}^{k}\left(\xi_{i}\right), y_{i}^{(k)}\right)=d_{i}\left(\varphi_{(N+1), i}\left(f_{N+1}^{k}\left(\xi_{N+1}\right)\right), \varphi_{(N+1), i}\left(y_{N+1}^{(k)}\right)\right)<\frac{\varepsilon}{2},
$$

所以

$$
d\left(f_{\infty}^{k}(\xi), \mathbb{y}_{k}\right) \leqslant \sum_{i=1}^{N+1} \frac{1}{2^{i}} \frac{d_{i}\left(f_{i}^{k}\left(\xi_{i}\right), y_{i}^{(k)}\right)}{1+d_{i}\left(f_{i}^{k}\left(\xi_{i}\right), y_{i}^{(k)}\right)}+\sum_{i=N+2}^{\infty} \frac{1}{2^{i}}<\sum_{i=1}^{N+1} \frac{1}{2^{i}} \frac{\varepsilon / 2}{1+\varepsilon / 2}+\frac{\varepsilon}{2}<\varepsilon .
$$


因此

$$
\bigcup_{n \in \mathbb{N}} B_{n}\left(\xi_{N+1}, \mathbb{Z}_{N+1}, f_{N+1}, \delta_{1}\right) \subset \bigcup_{n \in \mathbb{N}} B_{n}\left(\xi, \mathbb{Y}, f_{\infty}, \varepsilon\right)
$$

故而

$$
\limsup _{n \rightarrow \infty} \frac{1}{n}\left|B_{n}\left(\xi, \mathbb{Y}, f_{\infty}, \varepsilon\right)\right| \geqslant \limsup _{n \rightarrow \infty} \frac{1}{n}\left|B_{n}\left(\xi_{N+1}, \mathbb{Z}_{N+1}, f_{N+1}, \delta_{1}\right)\right|>\frac{1}{2} .
$$

由此和 $\varepsilon$ 的任意性可得 $f_{\infty}$ 具有 $\bar{d}$ - 跟踪性质.

通过定理 3.1 的证明过程, 同理可证以下定理成立.

定理 3.2 设 $\left(X_{\infty}, f_{\infty}\right)$ 为由 $\left\{\left(X_{i}, \varphi_{i}, f_{i}\right)\right\}_{i=1}^{\infty}$ 生成的逆极限系统. 如果每个 $f_{i}$ 具有 $\underline{d}$ - 跟踪性质 (或者, 遍历跟踪性质), 则 $f_{\infty}$ 具有 $\underline{d}$ - 跟踪性质 (相应地, 遍历跟踪性质).

\section{2 逆极限系统 $\left(X_{f}, \sigma_{f}\right)$ 的遍历伪轨跟踪性质}

1992 年, Chen 和 $\mathrm{Li}{ }^{[26]}$ 证明了 $\left(X_{f}, \sigma_{f}\right)$ 具有伪轨跟踪性质当且仅当 $(X, f)$ 具有伪轨跟踪性质. 最近, 我们在文献 $[27]$ 中得到了 $\left(X_{f}, \sigma_{f}\right)$ 是 $\left(\mathscr{F}_{1}, \mathscr{F}_{2}\right)$ - 混沌当且仅当 $(X, f)$ 是 $\left(\mathscr{F}_{1}, \mathscr{F}_{2}\right)$ - 混沌, 其中 $\mathscr{F}_{1}, \mathscr{F}_{2}$ 是平移不变的 Furstenberg 族. 受此启发, 本小节将进一步研究逆极限系统 $\left(X_{f}, \sigma_{f}\right)$ 的遍历伪 轨跟踪性质.

对任意 $A \subset \mathbb{Z}^{+}$, 定义 $A$ 的上密度和下密度分别为

$$
\overline{\operatorname{Dens}}(A)=\limsup _{n \rightarrow \infty} \frac{1}{n}|A \cap\{0,1, \ldots, n-1\}|, \quad \underline{\operatorname{Dens}}(A)=\liminf _{n \rightarrow \infty} \frac{1}{n}|A \cap\{0,1, \ldots, n-1\}| .
$$

若 $\overline{\operatorname{Dens}}(A)=\operatorname{Dens}(A)=\xi$, 则称 $A$ 的密度为 $\xi$, 记为 $\operatorname{Dens}(A)=\xi$.

下面的引理是自然成立的, 在此略去证明.

引理 3.3 假设 $A \subset \mathbb{Z}^{+}$满足 $\operatorname{Dens}(A)=0$. 则对任意 $N \in \mathbb{N}, \operatorname{Dens}\left(\bigcup_{j=0}^{N}(A-j)\right)=0$.

命题 3.4 假设 $f: X \longrightarrow X$ 为连续满射. 如果对任意 $\varepsilon>0$, 存在 $\delta>0$ 及 $p \in \mathbb{Z}^{+}$, 使得对 $f$ 的任意 $\delta$ - 遍历伪轨 $\left\{x_{i}\right\}_{i=0}^{\infty}$, 存在 $z \in X$ 满足 $\lim \sup _{n \rightarrow \infty} \frac{1}{n}\left|B_{n}\left(z,\left\{f^{p}\left(x_{i}\right)\right\}_{i=0}^{\infty}, f, \varepsilon\right)\right|>1 / 2$ (或者, $\left.\liminf _{n \rightarrow \infty} \frac{1}{n}\left|B_{n}\left(z,\left\{f^{p}\left(x_{i}\right)\right\}_{i=0}^{\infty}, f, \varepsilon\right)\right|>0, \lim _{n \rightarrow \infty} \frac{1}{n}\left|B_{n}\left(z,\left\{f^{p}\left(x_{i}\right)\right\}_{i=0}^{\infty}, f, \varepsilon\right)\right|=1\right)$, 则 $f$ 具有 $\bar{d}$ - 跟踪性 质 (相应地, $\underline{d}$ - 跟踪性质, 遍历跟踪性质).

证明 任意取定 $\varepsilon>0$, 则存在 $\beta \in(0, \varepsilon)$ 及 $P \in \mathbb{N}$, 使得对 $f$ 的任意 $\beta$ - 遍历伪轨 $\left\{x_{i}\right\}_{i=0}^{\infty}$, 存 在 $z \in X$ 满足 $\limsup _{n \rightarrow \infty} \frac{1}{n}\left|B_{n}\left(z,\left\{f^{P}\left(x_{i}\right)\right\}_{i=0}^{\infty}, f, \varepsilon / 2\right)\right|>1 / 2$. 由于 $f$ 一致连续, 所以存在 $0<\eta<$ $\beta /(2 P)$, 使得

$$
\text { 对任意 } x, y \in X \text { 满足 } d(x, y)<\eta \text { 及任意 } 0 \leqslant j \leqslant P \text {, 恒有 } d\left(f^{j}(x), f^{j}(y)\right)<\frac{\beta}{2 P} \text {. }
$$

任意取定 $f$ 的一条 $\eta$ - 遍历伪轨 $\left\{y_{i}\right\}_{i=0}^{\infty}$, 由已知条件得

$$
\text { 存在 } z_{0} \in X \text {, 使得 } \limsup _{n \rightarrow \infty} \frac{1}{n}\left|B_{n}\left(z_{0},\left\{f^{P}\left(y_{i}\right)\right\}_{i=0}^{\infty}, f, \varepsilon / 2\right)\right|>\frac{1}{2} \text {. }
$$

为证明叙述方便, 记

$$
\Xi:=\bigcup_{n \in \mathbb{N}} B_{n}\left(\left\{y_{i}\right\}_{i=0}^{\infty}, f, \eta\right), \text { 并且 } \Xi^{c}:=\mathbb{Z}^{+}-\Xi .
$$

由于 $\left\{y_{i}\right\}_{i=0}^{\infty}$ 为 $f$ 的 $\eta$ - 遍历伪轨, 由引理 3.3 得

$$
\operatorname{Dens}\left(\mathbb{Z}^{+}-\bigcup_{j=0}^{P}\left(\Xi^{c}-j\right)\right)=1-\operatorname{Dens}\left(\bigcup_{j=0}^{P}\left(\Xi^{c}-j\right)\right)=1 .
$$


类似于 (2.6), 应用 (3.10) 式不难证明

$$
\limsup _{n \rightarrow \infty} \frac{1}{n}\left|\Omega:=\left(\mathbb{Z}^{+}-\bigcup_{j=0}^{P}\left(\Xi^{c}-j\right)\right) \cap\left(\bigcup_{n \in \mathbb{N}} B_{n}\left(z_{0},\left\{f^{P}\left(y_{i}\right)\right\}_{i=0}^{\infty}, f, \varepsilon / 2\right)\right)\right|>\frac{1}{2} .
$$

任意取定 $k \in \Omega$, 由 $\Omega$ 的选取则有对任意 $0 \leqslant j \leqslant P, k+j \in \Xi$ 恒成立. 由此和 (3.9) 式得

$$
\begin{aligned}
d\left(f^{P}\left(y_{k}\right), y_{P+k}\right) \leqslant & d\left(f^{P}\left(y_{k}\right), f^{P-1}\left(y_{k+1}\right)\right)+d\left(f^{P-1}\left(y_{k+1}\right), f^{P-2}\left(y_{k+2}\right)\right) \\
& +\cdots+d\left(f\left(y_{P+k-1}\right), y_{P+k}\right) \\
\leqslant & P \cdot \frac{\beta}{2 P}<\frac{\varepsilon}{2} .
\end{aligned}
$$

这说明

$$
\begin{aligned}
d\left(f^{k}\left(z_{0}\right), y_{P+k}\right) & \leqslant d\left(f^{k}\left(z_{0}\right), f^{P}\left(y_{k}\right)\right)+d\left(f^{P}\left(y_{k}\right), y_{P+k}\right) \\
& <\frac{\varepsilon}{2}+\frac{\varepsilon}{2}=\varepsilon\left(\text { 因 } k \in \bigcup_{n \in \mathbb{N}} B_{n}\left(z_{0},\left\{f^{P}\left(y_{i}\right)\right\}_{i=0}^{\infty}, f, \frac{\varepsilon}{2}\right)\right) .
\end{aligned}
$$

取 $z^{*} \in f^{-P}\left(z_{0}\right)$ (因为 $f$ 满射, $z^{*}$ 是存在的). 由以上的讨论, 则不难得出对任意 $k \in \Omega$, 恒有

$$
d\left(f^{P+k}\left(z^{*}\right), y_{P+k}\right)=d\left(f^{k}\left(z_{0}\right), y_{P+k}\right)<\varepsilon,
$$

即 $\Omega+P \subset \bigcup_{n \in \mathbb{N}} B_{n}\left(z^{*},\left\{y_{i}\right\}_{i=0}^{\infty}, f, \varepsilon\right)$. 由此和 (3.12) 式及 $\varepsilon$ 的任意性可知 $f$ 具有 $\bar{d}$ - 伪轨跟踪性. 类 似于以上证明过程, 不难验证该命题对于 $\underline{d}$ - 跟踪性质和遍历跟踪性质均成立.

虽然现在仍未知定理 3.1 和 3.2 的逆命题是否成立, 但对于移位同胚 $\sigma_{f}$, 我们有如下定理:

定理 3.5 逆极限系统 $\left(X_{f}, \sigma_{f}\right)$ 具有 $\bar{d}$ - 跟踪性质 (或者, $\underline{d}$ - 跟踪性质, 遍历跟踪性质) 当且仅当 $(X, f)$ 具有 $\bar{d}$ - 跟踪性质 (或者, $\underline{d}$ - 跟踪性质, 遍历跟踪性质).

证明 由定理 3.1 和 3.2 知充分性是显然成立的. 下证必要性.

任意取定 $\varepsilon>0$, 由于 $\sigma_{f}$ 具有 $\bar{d}$ - 跟踪性质, 所以存在 $\delta \in(0, \varepsilon / 2)$, 使得对 $\sigma_{f}$ 的任意 $\delta$ - 遍历伪轨 $\left\{x_{i}\right\}_{i=0}^{\infty}$, 存在 $z \in X_{f}$ 满足 $\lim \sup _{n \rightarrow \infty} \frac{1}{n}\left|B_{n}\left(z,\left\{x_{i}\right\}_{i=0}^{\infty}, \sigma_{f}, \varepsilon / 2(1+\varepsilon)\right)\right|>1 / 2$. 取充分大的 $N \in \mathbb{N}$, 使 得 $\sum_{n=N+1}^{\infty} 1 / 2^{n}<\delta / 2$. 注意到 $f$ 一致连续, 则存在 $0<\xi<\delta / 2$, 使得任意 $x, y \in X$ 满足 $d(x, y)<\xi$ 及任意 $0 \leqslant j \leqslant N$, 恒有

$$
d\left(f^{j}(x), f^{j}(y)\right)<\frac{\delta}{2} .
$$

任取 $f$ 的 $\xi$ - 遍历伪轨 $\mathrm{y}:=\left\{y_{i}\right\}_{i=0}^{\infty}$, 同时令 $\mathrm{y}^{(i)}=\left(f^{N}\left(y_{i}\right), f^{N-1}\left(y_{i}\right), \ldots, y_{i}, \ldots\right) \in X_{f}$ (因 $f$ 满射, $\mathrm{y}^{(i)}$ 存在).

$$
\begin{array}{cccccc}
\left\{\mathrm{y}^{(i)}\right\}_{i=0}^{\infty} & f^{N}(\mathrm{y}) & f^{N-1}(\mathrm{y}) & \ldots & \mathrm{y} & \ldots \\
\mathrm{y}^{(0)} & f^{N}\left(y_{0}\right) & f^{N-1}\left(y_{0}\right) & \ldots & y_{0} & \ldots \\
\mathbb{y}^{(1)} & f^{N}\left(y_{1}\right) & f^{N-1}\left(y_{1}\right) & \ldots & y_{1} & \ldots \\
\vdots & \vdots & \vdots & & \vdots & \\
\mathrm{y}^{(n)} & f^{N}\left(y_{n}\right) & f^{N-1}\left(y_{n}\right) & \ldots & y_{n} & \ldots \\
\mathbb{y}^{(n+1)} & f^{N}\left(y_{n+1}\right) & f^{N-1}\left(y_{n+1}\right) & \ldots & y_{n+1} & \ldots
\end{array}
$$


对任意 $j \in \bigcup_{n \in \mathbb{N}} B_{n}(\mathrm{y}, f, \xi)$, 注意到

$$
\sigma_{f}\left(\mathbb{y}^{(j)}\right)=\left(f^{N+1}\left(y_{j}\right), f^{N}\left(y_{j}\right), \ldots, f\left(y_{j}\right), \ldots\right),
$$

所以由 $N$ 的选取及 (3.16) 式知

$$
\begin{aligned}
d_{\infty}\left(\sigma_{f}\left(\mathrm{y}^{(j)}\right), \mathbb{y}^{j+1}\right) & \leqslant \sum_{n=1}^{N+1} \frac{1}{2^{n}} \frac{d\left(f^{N+2-n}\left(y_{j}\right), f^{N+1-n}\left(y_{j+1}\right)\right)}{1+d\left(f^{N+2-n}\left(y_{j}\right), f^{N+1-n}\left(y_{j+1}\right)\right)}+\frac{\delta}{2} \\
& <\sum_{n=1}^{N+1} \frac{1}{2^{n}} \frac{\delta / 2}{1+\delta / 2}+\frac{\delta}{2}<\delta .
\end{aligned}
$$

这说明 $\left\{\mathrm{y}^{(i)}\right\}_{i=0}^{\infty}$ 为 $\sigma_{f}$ 的 $\delta$ - 遍历伪轨. 因此

$$
\text { 存在 } \mathbb{Z} \in X_{\infty} \text {, 使得 } \limsup _{n \rightarrow \infty} \frac{1}{n}\left|B_{n}\left(\mathbb{Z},\left\{\mathrm{y}^{(i)}\right\}_{i=0}^{\infty}, \sigma_{f}, \varepsilon / 2(1+\varepsilon)\right)\right|>\frac{1}{2} \text {. }
$$

由此不难证明

$$
\limsup _{n \rightarrow \infty} \frac{1}{n}\left|B_{n}\left(\pi_{1}(\mathbb{Z}),\left\{f^{N}\left(y_{i}\right)\right\}_{i=0}^{\infty}, f, \varepsilon\right)\right|>\frac{1}{2} .
$$

由命题 3.4 自然可得 $f$ 具有 $\bar{d}$ - 跟踪性质. 同理可证 $\underline{d}$ - 跟踪性质及遍历跟踪性质.

致谢 本文为作者在香港城市大学作学术访问期间完成的。同时, 作者对审稿人的有益建议深表感谢.

\section{参考文献}

1 Blank M L. Metric properties of $\varepsilon$-trajectory of dynamical systems with stochastic behavior. Ergodic Theory Dyn Syst, 1988, 8: 365-378

2 Eirola T, Nevanlinna O, Pilyugin S Y. Limit shadowing property. Numer Funct Anal Math, 1997, 18: 75-92

3 Gu R B. The asymptotic average shadowing property and transitivity. Nonlinear Anal, 2007, 67: 1680-1689

4 Fakhari A, Ghane F H. On shadowing: Ordinary and ergodic. J Math Anal Appl, 2010, 364: 151-155

5 Dastjerdi D A, Hosseini M. Sub-shadowings. Nonlinear Anal, 2010, 72: 3759-3766

6 Oprocha P, Dastjerdi D A, Hosseini M. On partial shadowing of complete pseudo-orbits. J Math Anal Appl, 2014, 411: 454-463

7 Bowen R. Equilibrium States and Ergodic Theory of Anosov Diffieomorphisms. Lecture Notes in Math. Berlin: Springer-Verlag, 1975

8 Barge M, Swanson R. Pseudo-orbits and topological entropy. Proc Amer Math Soc, 1990, 109: 559-566

9 Boyarskia A, Góra P. The pseudo-orbit shadowing property for Markov operators in the space of probability density functions. Canad J Math, 1990, 6: 1000-1017

10 Coven E M, Kan I, Yorke J A. Pseudo-orbit shadowing in the family of tent maps. Trans Amer Math Soc, 1988, 308: $227-241$

11 Walters P. On the pseudo-orbit tracing property and its relationship to stability. Lect Notes in Math, 668. BerlinHeidelbery: Springer-Verlag, 1978, 231-244

12 杨润生. 伪轨跟踪与混沌. 数学学报, 1996, 39: 382-386

13 杨润生. 伪轨跟踪与不变概率测度. 数学年刊, 1998, 19: 33-36

14 杨润生, 沈苏林. 伪轨跟踪与完全正熵. 数学学报, 1999, 42: 99-104

15 Richeson D, Wiseman J. Chain recurrence rates and topological entropy. Topology Appl, 2008, 156: 251-261

16 朱圣芝. 动力系统在链回复集上的拓扑稳定性. 中国科学: 数学, 2011, 41: 317-322

17 张筑生. 微分动力系统原理. 北京: 科学出版社, 1987

18 Kulczycki M, Kwietniak D, Oprocha P. On almost specification and average shadowing properties. Fund Math, 2014, 224: $241-278$

19 Aoki N, Hiraide K. Topological Theory of Dynamical Systems. North-Holland Mathematical Library, vol. 52. Amsterdam: North-Holland, 1994 
20 Kulczycki M, Oprocha P. Properties of dynamical systems with the asymptotic average shadowing properties. Fund Math, 2011, 212: 35-52

$21 \mathrm{Gu}$ R B. On ergodicity of systems with the asymptotic average shadowing property. Comput Math Appl, 2008, 55: 1137-1141

22 Auslander J, Yorke J A. Interval maps, factors of maps, and chaos. Tôhoku Math J, 1980, 32: 177-188

23 李思敏. 逆极限空间的伪轨跟踪性. 数学年刊, 2001, 22: 479-482

24 Gu R B, Shen Y Q. APOTP for the inverse limit spaces. Appl Math J Chin Univ Ser B, 2006, 21: 473-478

25 牛应轩. 具有渐近平均跟踪性质的系统. 高校应用数学学报, 2007, 22: 462-468

26 Chen L, Li S. Shadowing property for inverse limit spaces. Proc Amer Math Soc, 1992, 115: 573-580

27 吴新星, 朱培勇. 由双 Furstenberg 族诱导的混沌. 数学学报, 2012, 55: 1039-1054

\section{Some remarks on $\bar{d}$-shadowing property}

\section{WU XinXing}

Abstract $\quad \bar{d}$ - and $\underline{d}$-shadowing properties were introduced by Dastjerdi and Hosseini in 2010 for generalizing the pseudo-orbit shadowing property. The aim of this paper is to investigate $\bar{d}$ - and $\underline{d}$-shadowing properties of iteration systems and inverse limit systems. First, it is proved that for a dynamical system, the following three statements are equivalent: (1) $f$ has $\bar{d}$-shadowing property (resp., $\underline{d}$-shadowing property); (2) $f^{k}$ has $\bar{d}$-shadowing property (resp., $\underline{d}$-shadowing property) for all $k \in \mathbb{N} ;(3) f^{k}$ has $\bar{d}$-shadowing property (resp., $\underline{d}$-shadowing property) for some $k \in \mathbb{N}$. Moreover, it is proved that a dynamical system having $\bar{d}$-shadowing property is chain mixing. Finally, we obtain that the inverse limit system $\left(X_{\infty}, f_{\infty}\right)$ generated by $\left\{X_{i}, \varphi_{i}, f_{i}\right\}_{i=1}^{\infty}$ has $\bar{d}$-shadowing property (resp., $\underline{d}$-shadowing property, ergodic shadowing property) provided that every $f_{i}$ has $\bar{d}$-shadowing property (resp., $\underline{d}$-shadowing property, ergodic shadowing property).

Keywords $\bar{d}$-shadowing property, $\underline{d}$-shadowing property, chain mixing, inverse limit system

MSC(2010) 54H20, 37B40, 37D45, 28D20

doi: 10.1360/N012013-00171 